\title{
EFECTO DE PLANTACIONES DENDROENERGÉTICAS EN EL CARBONO A NIVEL DE SUELO, EN DOS SUELOS CONTRASTANTES DE LA REGIÓN DE BIOBÍO, CHILE ${ }^{1}$
}

\author{
Edwin Esquivel ${ }^{2}$, Rafael Rubilar ${ }^{3}$, Simón Sandoval ${ }^{4}$, Eduardo Acuña $^{4}$, Jorge Cancino ${ }^{4}$, Miguel Espinosa ${ }^{4}$ \\ e Fernando Muñoz ${ }^{4}$
}

\begin{abstract}
RESUMEN - La biomasa forestal como fuente de energía proveniente de plantaciones dendroenergéticas, al compararla con combustibles fósiles, presenta la ventaja de producir energía carbono-neutral, dado el secuestro de carbono (C) fijado en la biomasa producida y los aportes al suelo. Plantaciones forestales de corta rotación, altas densidades, y localizadas en terrenos marginales pueden representar una excelente oportunidad de producción de biomasa para la producción de energía o combustibles. Sin embargo, los beneficios en el corto plazo del aumento del C a nivel de suelo en sitios marginales han sido cuestionados. Se establecieron plantaciones dendroenergéticas con las especies Eucalyptus camaldulensis, E. nitens, E. globulus, y Acacia melanoxylon a densidades de 5.000, 7.500 y 10.000 plantas por hectárea, en dos sitios de producción forestal marginal con suelos contrastantes (arenales y granítico) localizados en la Región del Biobío de Chile. Evaluaciones periódicas durante 4 años a 0-20 y 20-40 cm de profundidad, del nivel de C en cada uno de los suelos evaluados, sugiere efectos de la edad (tiempo) desde el establecimiento de la plantación $(P<0,001)$. A pesar de los cambios observados en el tiempo, la escasa diferencia en los niveles observados de $C$ en el suelo mineral para los 48 meses de estudio comparados a los primeros 2 meses post-plantación, sugiere una rápida capacidad de recuperación de los niveles de C del suelo en un corto periodo.
\end{abstract}

Palabras-clave: Bioenergía; Sustentabilidad; Plantaciones de alta densidad.

\section{EFEITO DE PLANTAÇÕES DENDROENERGÉTICAS NO CARBONO AO NÍVEL DO SOLO, EM DOIS SOLOS CONTRASTANTES DA REGIÃO DE BIOBÍO, CHILE}

\begin{abstract}
RESUMO - A biomassa florestal para energia a partir de plantios de madeira para energia, quando comparada com os combustíveis fósseis, tem a vantagem de produzir carbono neutro de energia, como o sequestro de carbono (C) ligado à biomassa produzida e contribuições para o solo. Plantios florestais localizados em sítios marginais de curta rotação e densidades elevadas podem constituir excelente oportunidade para a produção de biomassa para energia ou combustíveis. No entanto, os benefícios no curto prazo de aumento do C em nível do solo em sítios marginais têm sido questionados. Plantios energéticos de espécies madeireiras foram estabelecidos com Eucalyptus camaldulensis, E. nitens, E. globulus e Acacia melanoxylon, com densidades de 5.000, 7.500 e 10.000 plantas por hectare, em duas áreas de floresta de produção marginais com solos contrastantes (areias e granito), localizado na Região do Biobío, no Chile. Avaliações periódicas para quatro anos, em profundidades de 0-20 cm e 20-40 do nível de C em cada um dos solos testados, sugerem efeitos da idade (tempo) desde o estabelecimento do plantio $(P<0,001)$. Além das alterações observadas no tempo, a diferença pouco significativa nos níveis de C no solo mineral em 48 meses desde o início do estudo, em comparação com os primeiros dois meses pós-plantio, sugere uma capacidade de rápida recuperação dos níveis de C em períodos curtos.
\end{abstract}

Palavras-chave: Bio-energia; Sustentabilidade; Plantações de alta densidade.

\footnotetext{
${ }^{1}$ Recebido em 07.12.2012 aceito para publicação em 23.10.2013.

${ }^{2}$ Escuela de Ingeniería Forestal Instituto Tecnológico de Costa Rica. E-mail:<eesquivel@itcr.ac.cr>.

${ }^{3}$ Cooperativa de Productividad Forestal, Facultad de Ciencias Forestales, Universidad de Concepción, Chile. E-mail: <rrubilar@ncsfnc.cfr.ncsu.edu>.

${ }^{4}$ Facultad de Ciencias Forestales, Universidad de Concepción, Chile. E-mail:<sisandoval@udec.cl>, <edacuna@udec.cl>, <jcancino@udec.cl>,<mespinos@udec.cl>e < fmunoz@udec.cl>.
} 


\section{INTRODUCCIÓN}

La mayor fracción de las emisiones de $\mathrm{CO}_{2}$ atmosférico es producto de la quema de combustibles fósiles, que actualmente satisfacen aproximadamente el 85\% del consumo energético mundial. Estas emisiones podría reducirse mediante la utilización de la biomasa, la cual tiene el potencial de convertirse en la mayor fuente primaria de energía del próximo siglo (BERNDES et al., 2003), y entre las principales fuentes de biomasa, las plantaciones dendroenergéticas son una opción atractiva dada su adaptabilidad (KARP; SHIELD, 2008) y su posibilidad de satisfacer las crecientes necesidades energéticas tanto con combustibles sólidos y líquidos (CARROLL; SOMERVILLE, 2009).

Se ha identificado que una de las grandes ventajas de las plantaciones dendroenergéticas es que logran aumentar los reservorios de carbono no atmosférico mediante tres vías: la primera, mediante el aumento del secuestro de carbono fijándolo en la biomasa y en el suelo (SARTORI et al., 2006); la segunda, mediante el mantenimiento de carbono ya secuestrado (ERICSSON, 2003), y la tercera, mediante la sustitución de combustibles fósiles y carbón mineral (BARAL; GUHA, 2004). Las dos primeras permiten una distinción de la biomasa con las otras fuentes de bioenergía (BERNDES et al., 2003). Los cultivos dendroenergéticos, y en especial los lignocelulósicos, presentan mayores potenciales de reducción de gases con efecto invernadero (ADLER et al., 2007), pudiendo llegar estas a ser eventualmente carbono neutrales (ANDERSEN et al., 2005; FUNG et al., 2002).

Se ha observado que el manejo que recibe el cultivo tiene relación directa con la magnitud de la reducción potencial de emisiones (ADLER et al., 2007) y con otros efectos favorables en el ecosistema. Plantaciones dendroenergéticas establecidas en suelos tradicionalmente agrícolas han aumentado los niveles de materia orgánica del suelo, la calidad de agua, la microflora, la microfauna y la porosidad del suelo; con una consecuente disminución de la compactación (MANN; TOLBERT, 2000). Sin embargo, se ha observado que la remoción de residuos o árboles completos, afecta negativamente los rendimientos (MAFRA et al., 2008) de plantaciones en el corto y mediano plazo (5-30 años), en áreas donde el crecimiento es limitado por nitrógeno (STUPAK et al., 2007) o en sitios con reservas bajas de nutrientes (MAFRA et al., 2008). Debe considerarse también, que en estos cultivos, se han observado altas correlaciones entre los reservorios de $\mathrm{N}$ del suelo y los niveles de $\mathrm{C}$ entre los 0 y $30 \mathrm{~cm}$ de profundidad (SARTORI et al., 2007).

La captura y mantenimiento de los niveles de carbono en el de suelo dependen de factores como: uso histórico, tipo de suelo, especie, ciclo de cosechas y otras prácticas de manejo como fertilización (BENITES et al., 2010; SARTORI et al., 2006). Sin embargo, existen evidencias contrastantes sobre los efectos de las plantaciones dendroenergéticas en los niveles de carbono del suelo. Se ha evidenciado que los niveles de carbono precosecha se mantienen durante el primer año atribuyéndolo a la rápida incorporación de la necromasa, evidenciando disminuciones hasta los 3 años postcosecha (SANCHEZ et al., 2007). Otras investigaciones encontraron diferencias en los años iníciales en los niveles de carbono de suelo, encontrando pequeños incrementos hasta los 40 meses de la plantación (COLEMAN et al., 2004).

Con el objetivo de evaluar la dinámica de carbono en plantaciones dendroenergéticas, se estableció este ensayo bajo la hipótesis de que la cantidad de carbono en el suelo se ve afectada por el sitio y es influenciada por la especie plantada y la densidad de plantación, y el efecto puede evaluarse en un plazo menor a 4 años. Teniéndose que especies fijadoras de nitrógeno presentarían menores contenidos de carbono en de suelo que las especies no fijadoras y que la cantidad de carbono de suelo es directamente proporcional a la densidad de plantación en una especie determinada.

\section{MÉTODOS}

\subsection{Características y ubicación de los ensayos}

Se establecieron dos ensayos en agosto de 2007, en predios con rendimiento forestal marginal, ambos ubicados en la Región del Biobío, Chile; uno de ellos ubicado en la comuna de Ninhue $\left(36^{\circ} 17^{\prime}\right.$ Lat Sur, $72^{\circ} 22^{\prime}$ Long Oeste), con una topografía ondulada, el suelo es de origen granítico y pertenece a la serie Cauquenes (Xeralfs), caracterizado por su susceptibilidad a la erosión. La precipitación anual es de 700 mm, con temperaturas promedio anual de $13,2^{\circ} \mathrm{C}$. El terreno en el área del ensayo posee una pendiente no superior a $5 \%$. El otro predio, localizado en la comuna de Yungay (3703' Lat Sur, $72^{\circ} 11^{\prime}$ Long Oeste), con una topografía plana, el suelo es de origen andesítico basáltico y

Revista Árvore, Viçosa-MG, v.37, n.6, p.1135-1144, 2013

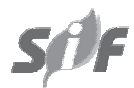


pertenece a la serie Arenales (Psamments); caracterizado por un drenaje excesivamente alto y por concentraciones bajas materia orgánica en todo el perfil. La precipitación anual es de $1.100 \mathrm{~mm}$. La temperatura promedio anual de $13^{\circ} \mathrm{C}$. El terreno en el área del ensayo posee un pendiente plana no superior al $1 \%$.

En los predios, el uso previo correspondió a plantaciones de Pinus radiata D. Don de 20 años de edad en el sitio arenales y 22 años de edad sitio granítico. Posterior a la cosecha de los rodales, se procedió a la remoción de tocones y residuos remanentes, para dar paso a una preparación de suelo con subsolado a $80 \mathrm{~cm}$ de profundidad, mediante un Caterpillar D8K, a un distanciamiento de $60 \mathrm{~cm}$ entre líneas. El subsolado fue realizado en dos direcciones perpendiculares formando un cuadriculado en el terreno. Un control de malezas pre-plantación fue aplicado a superficie completa, mediante una mezcla compuesta por $4 \mathrm{~kg} \mathrm{ha}^{-1}$ de GlifosatoRoundap Max y 1,5 kg ha-1 de Simazina (Granítico) y 2,5 $\mathrm{kg} \mathrm{ha}^{-1}$ de Atrazina (Arenales).

Los ensayos se establecieron con un diseño de bloques completos al azar con 3 réplicas. Los bloques fueron cuadrados de $75 \mathrm{~m}$ de lado $\left(5.625 \mathrm{~m}^{2}\right)$, estuvieron conformados por nueve unidades experimentales de $25 \mathrm{~m}$ de lado $\left(625 \mathrm{~m}^{2}\right.$ ), que incluían una zona de amortiguamiento de al menos 5 metros y una parcela núcleo central de medición. Los tratamientos consideraron densidades nominales de plantación de 5.000 árboles ha ${ }^{-1}(1,41$ x 1,41 m), 7.500 árboles ha-1 (1,15 x 1,15 m) y 10.000 árboles ha-1 $(1,00$ x 1,00 m) y tres especies por sitio. Las especies evaluadas en el sitio granítico fueron: Acacia melanoxylon, Eucalyptus camaldulensis y E. nitens; y en el sitio arenales se consideró: A. melanoxylon, E. globulus y E. nitens.

El crecimiento de los árboles fue evaluado en un núcleo central en cada unidad de muestreo. En el sitio granítico este núcleo fue de 49 árboles y, en el sitio arenales, para evaluar el rebrote, se realizaron parcelas divididas. Cada subparcela está constituida por un núcleo de 45 árboles para E. camaldulensis y E. nitens y en el caso de $A$. melanoxylon los núcleos fueron de 15 árboles para la densidad de 5.000 árboles ha1, 24 árboles para la densidad de 7.500 árboles ha-1 y 30 árboles para la densidad de 10.000 árboles ha ${ }^{-1}$.

La fertilización post-plantación se realizó a 25 cm del tallo de cada planta, utilizando en el predio granítico 30 g de Boronatrocalcita, 150 g de Fosfato Diamónico y 50 g de Sulpomag. En el predio arenales, la fertilización consistió en 25 g de Boronatrocalcita, 100 g de Fosfato Diamónico y 50 g de Sulpomag. A los 22 meses se realizó un control de malezas manual sin retirar los residuos del sitio; y los 30 meses se realizó una segunda fertilización con $120 \mathrm{~kg} \mathrm{ha}^{-1}$ de nitrógeno y $30 \mathrm{~kg} \mathrm{ha}^{-1}$ de boro.

\subsection{Evaluaciones realizadas}

Se realizaron evaluaciones de $\mathrm{C}$ en el suelo mineral previo a la preparación de terreno y a los 2, 4, 11, 16, 23, 28, 36, 40 y 48 meses de edad, mediante un muestreo compuesto (8 puntos de muestreo). En el segundo mes, las muestras fueron obtenidas desde los 0 a $20 \mathrm{~cm}$ de profundidad y, a partir del cuarto mes, las muestras fueron obtenidas a las profundidades de 0 a 20 y de 20 a $40 \mathrm{~cm}$. Las muestras fueron transportadas al laboratorio y secadas al aire por al menos 24 horas. La muestras fueron posteriormente tamizadas a $2 \mathrm{~mm}$ (mesh ATMS No10) determinando los porcentajes de suelo, grava y biomasa en la muestra.

La determinación de materia orgánica en el suelo fue realizada mediante el método de pérdida de peso por ignición WLOI (Weight-Loss-On-Ignition), (MAGDOFF, 1996). Para ello, una alícuota de $10 \mathrm{~g}$ de suelo ( $\pm 0,01 \mathrm{~g}$ ) fue secada por 24 horas en horno a $105^{\circ} \mathrm{C}$ para obtener el peso seco del suelo ( $\pm 0,01 \mathrm{~g}$ ). Posteriormente, las muestras fueron calcinadas en una mufla a $450^{\circ} \mathrm{C}$ por 24 horas y pesadas $( \pm 0,01 \mathrm{~g}$ ) para obtener el peso calcinado. Previo al pesaje, se permitió que las muestras alcanzaran temperatura ambiente en un desecador. Se realizó un replica cada 10 muestras como medida de control del procedimiento. El contenido de materia orgánica (MO \%) se determinó utilizando la fórmula:

$$
\operatorname{MO}(\%)=\frac{\mathrm{PSS}_{105^{\circ} \mathrm{C}}-\mathrm{PSC}_{450^{\circ} \mathrm{C}}}{\mathrm{PSS}_{105^{\circ} \mathrm{C}}} * 100
$$

donde:

PSS $_{105{ }^{\circ} \mathrm{C}}=$ peso seco del suelo (g)

$\mathrm{PSS}_{450{ }^{\circ} \mathrm{C}}=$ peso calcinado del suelo $(\mathrm{g})$.

Las determinaciones de carbono en suelo se escalaron utilizando el porcentaje de materia orgánica mediante la fórmula [2] acorde a (TABATABAI, 1996).

$$
\text { Carbono }=\frac{\mathrm{VS} * \mathrm{Da} * \mathrm{MO} \%}{2}
$$

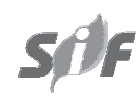

Revista Árvore, Viçosa-MG, v.37, n.6, p.1135-1144, 2013 
donde:

VS: Volumen de suelo $\left(\mathrm{m}^{3} \mathrm{ha}^{-1}\right)$

Da: Densidad aparente $\left(\mathrm{Mg} \mathrm{m}^{-3}\right)$

MO: Materia orgánica (\%).

La densidad aparente (Da) se determinó mediante muestreo por unidad experimental a profundidades de 0 a $20 \mathrm{~cm}$ y 20 a $40 \mathrm{~cm}$, tomadas con un barreno para muestreo de Da con cilindros de $5 \mathrm{~cm}$ de diámetro. Determinando el volumen del cada cilindro $( \pm 0,01 \mathrm{~mm})$ y el peso seco del suelo $( \pm 0,1 \mathrm{~g})$, para así poder utilizar la fórmula:

$$
\mathrm{Da}=\frac{P S S_{105^{\circ} \mathrm{C}}}{V c}
$$

donde:

$\mathrm{PSS}_{105{ }^{\circ} \mathrm{C}}=$ peso seco del suelo (g)

$\mathrm{Vc}=$ Volumen del cilindro $\left(\mathrm{cm}^{3}\right)$.

\subsection{Análisis estadístico de los datos obtenidos}

Los datos se sometieron a un análisis de homocedasticidad, mediante la prueba de Bartlett a los residuales del carbono de suelo y se aplicó la prueba de Shapiro-Wilks para verificar su normalidad, utilizando el software estadístico SAS Versión 9.1. Las medias se compararon utilizando la prueba de Tukey, con un 95\% de confianza, usando en la comparación de medias con modelos mixtos y análisis longitudinal en el programa SAS Versión 9.1 (SAS-INSTITUTE-INC, 2000).

\section{RESULTADOS}

Los análisis fueron realizados por sitio y por profundidad. En el sitio granítico, para la profundidad 0-20 cm, no se evidenció interacciones triples de los factores: especie, densidad y edad $(P=0,944)$. Se evidenció interacción entre especie y densidad $(P<0,001)$. La edad fue un factor significativo $(P<0,001)$ evidenciando cambios en el tiempo en los niveles de carbono del suelo mineral. Se evidenció el efecto de la densidad $(P<0,001)$ y de la especie $(P<0,001)$. En este sitio, a los 48 meses, presenta el contenido más alto de carbono $E$. camaldulensis con la densidad 5.000 árboles ha ${ }^{-1}$, sin que se evidenciara una diferencia con las densidades de 7.500 y 10.000 árboles ha-1 de la misma especie, así como tampoco logra una diferencia de A. melanoxylon a 5000 y 7500 árboles ha-1 ${ }^{-1}$ de $E$. nitens con la densidad de 7.500 árboles ha-1. Se destaca que los efectos encontrados previamente no han sido permanentes en el tiempo entre las diferentes edades de medición, Tabla 1 y 2. Como se puede observar en la Figura 1, existen diferencias entre los niveles de carbono inicial (línea base) y los niveles de carbono que ha mantenido el suelo durante los 48 meses; sin embargo, no existen evidencias de diferencias entre los niveles de carbono de los 2 meses y los 48 meses.

En la profundidad de 20-40 cm no se evidenció interacciones triples de los factores: especie, densidad y edad ( $P=0,512)$, igualmente la edad fue un factor significativo $(P<0,001)$ evidenciando cambios en el tiempo en los niveles de carbono del suelo a esa profundidad. Además, se evidenció efectos de la densidad $(P=0,003)$ en la cantidad de carbono del suelo, Tabla 1 . La densidad con mayor acumulación de carbono a nivel de suelo fue la de 7.500 árboles $\mathrm{ha}^{-1}$, sin diferencia con la de 5.000 árboles ha ${ }^{-1}$ Tabla 1 y 2 . Como se puede observar en la Figura 1, los niveles de carbono han descendido al compararlos con el contenido inicial (línea base); sin embargo, no existen evidencias de diferencias entre los niveles de los 2 meses y los 48 meses.

El sitio arenales, para la profundidad 0-20 cm, no se evidenció interacciones triples de los factores: especie, densidad y edad ( $P=0,513)$. También la edad fue un factor significativo $(P<0,001)$ evidenciando cambios en el tiempo en los niveles de carbono del suelo mineral. Se evidencian interacciones de la especie con la edad $(P=0,039)$ y de la densidad con la edad $(P=0,017)$; sin embargo, a los 48 meses no se observaron diferencias entre los tratamientos, Tabla 1 y 3. En la Figura 2, se puede apreciar que, a pesar del menor contenido de carbono con respecto al nivel previo al establecimiento, los niveles de carbono no difieren entre los 2 y 48 meses.

En la profundidad de $20-40 \mathrm{~cm}$, no se evidenció interacciones triples de los factores: especie, densidad y edad ( $P=0,302)$, igualmente la edad fue un factor significativo $(P<0,001)$ evidenciando cambios en el tiempo en los niveles de carbono del suelo. En esta profundidad, se evidenció el efecto de la especie $(P=0,011)$ en los niveles de carbono a nivel de suelo, sin embargo, a los 48 meses no se observaron diferencias entre los tratamientos, Tabla 1 y 3 . En la Figura 2, se aprecia un mayor contenido de carbono durante el periodo 
Tabla 1 - Valores p del análisis de varianza longitudinal del efecto de los tratamientos y sus interacciones en la concentración de carbono en suelo

Tabela 1 - Os valores de p da análise de variância longitudinal do efeito dos tratamentos e as suas interações na concentração de carbono no solo

\begin{tabular}{lcccccccc}
\hline Sitio & Profundidad & Especie (Sp) & Densidad (Den) & Sp*Den & Edad & SP*Edad & Den*Edad & SP*Den*Edad \\
\hline Granítico & $0-20$ & $<0,001$ & $<0,001$ & $<0,001$ & $<0,001$ & 0,897 & 0,527 & 0,944 \\
& $20-40$ & 0,229 & 0,003 & 0,128 & $<0,001$ & 0,292 & 0,353 & 0,512 \\
Arenales & $0-20$ & 0,595 & 0,242 & 0,979 & $<0,001$ & 0,039 & 0,017 & 0,513 \\
& $20-40$ & 0,011 & 0,251 & 0,604 & $<0,001$ & 0,768 & 0,248 & 0,302 \\
\hline
\end{tabular}

Tabla 2 - Promedio de carbono $\left(\mathrm{Mg} \mathrm{ha}^{-1}\right.$ ) a nivel de suelo para todas la especies (Acacia melanoxylon, Eucalyptus camaldulensis y E. nitens) en las todas las densidades (5.000, 7.500 y 10.000 árboles ha ${ }^{-1}$ ) en el sitio con suelo granítico a las profundidades 0-20 y 20-40 cm para cada edad evaluada.

Tabela 2 - Média de carbono (Mg ha ${ }^{-1}$ ) em nível do solo em todas as espécies (Acacia melanoxylon, Eucalyptus camaldulensis e E. nitens) em todas as densidades (5.000, 7.500 e 10.000 árvores ha ${ }^{-1}$ ), no local com um solo granítico às profundidades de 0-20 e 20-40 cm para cada idade avaliada.

\begin{tabular}{|c|c|c|c|c|c|c|c|c|c|c|}
\hline \multirow{2}{*}{ Especie } & \multirow{2}{*}{ Densidad } & \multicolumn{9}{|c|}{ Edad (meses) } \\
\hline & & 2 & 4 & 11 & 16 & 23 & 28 & 36 & 40 & 48 \\
\hline \multicolumn{11}{|c|}{ Profundidad $0-20 \mathrm{~cm}$} \\
\hline \multirow[t]{3}{*}{ A. melanoxylon } & 5.000 & $50,5 \mathrm{~ns}$ & $44,1 \mathrm{ab}$ & $47,7 \mathrm{~ns}$ & $54,5 \mathrm{ab}$ & $49,7 \mathrm{ab}$ & $43,8 \mathrm{~ns}$ & 47,8 a & 45,3 ns & $41,8 \mathrm{ab}$ \\
\hline & 7.500 & $43,7 \mathrm{~ns}$ & 53,4 a & $51,5 \mathrm{~ns}$ & $57,0 \mathrm{ab}$ & $50,3 \mathrm{ab}$ & $36,5 \mathrm{~ns}$ & $42,8 \mathrm{ab}$ & $49,8 \mathrm{~ns}$ & $41,5 \mathrm{ab}$ \\
\hline & 10.000 & $40,7 \mathrm{~ns}$ & $48,1 \mathrm{ab}$ & $46,7 \mathrm{~ns}$ & $50,8 \mathrm{ab}$ & $51,1 \mathrm{ab}$ & $36,6 \mathrm{~ns}$ & $43,4 \mathrm{ab}$ & $48,2 \mathrm{~ns}$ & $36,4 \mathrm{~b}$ \\
\hline \multirow[t]{3}{*}{ E. camaldulensis } & 5.000 & $55,3 \mathrm{~ns}$ & 53,5 a & $47,7 \mathrm{~ns}$ & 65,4 a & $54,5 \mathrm{ab}$ & $46,5 \mathrm{~ns}$ & $48,2 \mathrm{a}$ & 49,9 ns & 49,3 a \\
\hline & 7.500 & 47,4 ns & $49,4 \mathrm{ab}$ & $48,0 \mathrm{~ns}$ & $46,0 \mathrm{~b}$ & $42,1 \mathrm{ab}$ & $38,9 \mathrm{~ns}$ & $32,7 \mathrm{~b}$ & $46,3 \mathrm{~ns}$ & $41,5 \mathrm{ab}$ \\
\hline & 10.000 & $54,6 \mathrm{~ns}$ & $41,9 \mathrm{ab}$ & $49,8 \mathrm{~ns}$ & $53,8 \mathrm{ab}$ & 60,0 a & $36,4 \mathrm{~ns}$ & 51,4 a & $46,2 \mathrm{~ns}$ & $38,1 \mathrm{ab}$ \\
\hline \multirow[t]{3}{*}{ E. nitens } & 5.000 & $44,4 \mathrm{~ns}$ & $36,1 \mathrm{~b}$ & $42,3 \mathrm{~ns}$ & $47,8 \mathrm{~b}$ & $46,1 \mathrm{ab}$ & $36,1 \mathrm{~ns}$ & $37,7 \mathrm{ab}$ & $42,7 \mathrm{~ns}$ & $30,7 \mathrm{~b}$ \\
\hline & 7.500 & $50,8 \mathrm{~ns}$ & 45,3 ab & $55,2 \mathrm{~ns}$ & $45,4 \mathrm{~b}$ & $57,8 \mathrm{ab}$ & $52,6 \mathrm{~ns}$ & $42,6 \mathrm{ab}$ & $51,1 \mathrm{~ns}$ & $42,3 \mathrm{ab}$ \\
\hline & 10.000 & $39,9 \mathrm{~ns}$ & $39,1 \mathrm{ab}$ & $45,6 \mathrm{~ns}$ & $46,4 \mathrm{~b}$ & 39,9 b & $33,0 \mathrm{~ns}$ & $38,5 \mathrm{ab}$ & $36,5 \mathrm{~ns}$ & $32,6 \quad b$ \\
\hline \multicolumn{11}{|c|}{ Profundidad $20-40 \mathrm{~cm}$} \\
\hline & 5.000 & & $45,6 \mathrm{~ns}$ & $46,8 \mathrm{~ns}$ & $50,3 \mathrm{~ns}$ & $44,8 \mathrm{~b}$ & $35,9 \mathrm{~ns}$ & 42,9 ns & $48,3 \mathrm{~ns}$ & $37,1 \mathrm{ab}$ \\
\hline & 7.500 & & $50,0 \mathrm{~ns}$ & $53,0 \mathrm{~ns}$ & $49,2 \mathrm{~ns}$ & 55,1 a & $43,6 \mathrm{~ns}$ & $46,8 \mathrm{~ns}$ & $51,9 \mathrm{~ns}$ & 44,0 a \\
\hline & 10.000 & & $41,7 \mathrm{~ns}$ & $42,1 \mathrm{~ns}$ & $50,9 \mathrm{~ns}$ & $43,0 \mathrm{~b}$ & $35,2 \mathrm{~ns}$ & $41,1 \mathrm{~ns}$ & $41,8 \mathrm{~ns}$ & $33,6 \mathrm{~b}$ \\
\hline
\end{tabular}

Letras distintas indican diferencias significativas Test de Tukey, $\alpha=0,05$.

Letras diferentes indicam diferença significativa pelo teste de Tukey, $\alpha=0,05$.

evaluado que el muestreado antes de establecer del ensayo; sin embargo, no se han logrado evidenciar diferencias entre los 2 y los 48 meses en los niveles de carbono.

\section{DISCUSIÓN}

Tras la cosecha de la plantación forestal, se han evidenciado descensos en los niveles de carbono de suelo que pueden llegar a un 50\% del contenido precosecha. En este caso, se evidenció un descenso en los niveles de carbono entre la evaluación previa al establecimiento de los ensayos y los 2 meses de establecidos. Este es un efecto ampliamente documentado, el cual puede ocurrir en durante los primeros años post- cosecha; Yanai et al. (2011) sugieren que múltiples factores podrían estar correlacionados con los cambios en el suelo, los cuales pueden atribuirse a un efecto directo o indirecto post-cosecha en la descomposición del carbono lábil del suelo. MERINO et al. (1998); USSIRI y JOHNSON (2007) concuerdan con el descenso postcosecha de la materia orgánica del suelo y, en consecuencia, del carbono; atribuyen este efecto a cambios en la calidad de la materia orgánica.

La disminución del carbono en el suelo en los primeros meses puede ser atribuida a la descomposición de las raíces finas y micorrizas (POWERS et al., 2005), que dejan de cumplir su función metabólica y, por ende, de aportar carbono al suelo; además, se descomponen

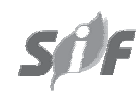

Revista Árvore, Viçosa-MG, v.37, n.6, p.1135-1144, 2013 

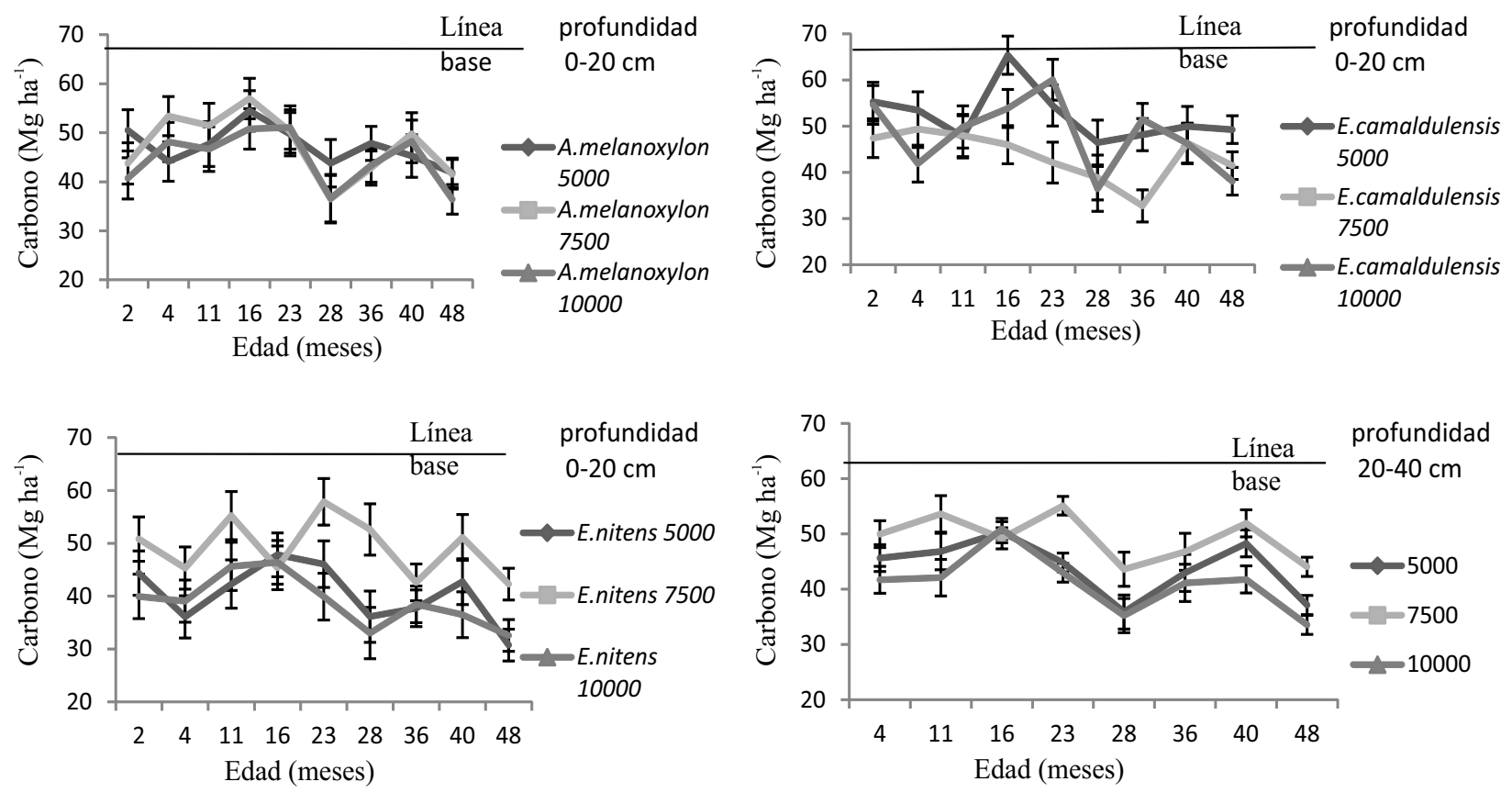

Figura 1 - Promedios para los efectos significativos de contenido de carbono ( $\left.\mathrm{Mg} \mathrm{ha}^{-1}\right)$ para todas la especies (Acacia melanoxylon, Eucalyptus camaldulensis y E. nitens) en todas las densidades (5.000, 7.500 y 10.000 árboles ha-1) a las profundidades 0-20 y 20-40 cm, en el sitio de suelos graníticos. Barras de error corresponden al error estándar.

Figura 1 - Valores médios dos efeitos significativos do conteúdo de carbono $\left(\mathrm{Mg} \mathrm{ha}^{-1}\right)$ em todas as espécies (Acacia melanoxylon, Eucalyptus camaldulensis e E. nitens) em todas as densidades (5.000, 7.500 e 10.000 árvores ha ${ }^{-1}$ ), às profundidades de 0-20 e 20-40 cm, no local de solos graníticos. As barras de erro correspondem ao erropadrão.

Tabla 3 - Promedio de carbono $\left(\mathrm{Mg} \mathrm{ha}^{-1}\right)$ en nivel de suelos para todas las densidades (5.000, 7.500 y 10.000 árboles ha-1) y para todas la especies (Acacia melanoxylon, Eucalyptus globulus y E. nitens) en el sitio con suelo arenales a las profundidades 0-20 y 20-40 cm para cada edad evaluada.

Tabela 3 - Média de carbono ( $\mathrm{Mg} \mathrm{ha}^{-1}$ ) no nível do solo para todas as densidades (5.000, 7.500 e 10.000 árvores ha-1), em todas as espécies (Acacia melanoxylon, Eucalyptus globulus e E. nitens), no local com um solo arenoso às profundidades 0-20 e 20-40 cm para cada idade avaliada.

\begin{tabular}{|c|c|c|c|c|c|c|c|c|c|}
\hline \multirow{2}{*}{ Especie o Densidad } & \multicolumn{9}{|c|}{ Edad (meses) } \\
\hline & 2 & 4 & 11 & 16 & 23 & 28 & 36 & 40 & 48 \\
\hline \multicolumn{10}{|c|}{ Profundidad $0-20 \mathrm{~cm}$} \\
\hline A. melanoxylon & $30,2 \mathrm{~ns}$ & $23,3 \mathrm{~ns}$ & $25,0 \quad b$ & $27,6 \mathrm{~ns}$ & $27,1 \mathrm{~ns}$ & $25,0 \mathrm{~ns}$ & $26,9 \mathrm{~ns}$ & $28,0 \mathrm{~ns}$ & $26,6 \mathrm{~ns}$ \\
\hline E. globulus & $27,5 \mathrm{~ns}$ & 23,7 ns & $36,1 \quad a$ & 30,9 ns & $26,1 \mathrm{~ns}$ & 27,6 ns & $26,1 \mathrm{~ns}$ & $25,4 \mathrm{~ns}$ & $27,7 \mathrm{~ns}$ \\
\hline E. nitens & $27,5 \mathrm{~ns}$ & $21,9 \mathrm{~ns}$ & $32,8 \mathrm{ab}$ & $28,3 \mathrm{~ns}$ & $30,2 \mathrm{~ns}$ & $28,0 \mathrm{~ns}$ & $30,3 \mathrm{~ns}$ & $30,0 \mathrm{~ns}$ & $32,0 \mathrm{~ns}$ \\
\hline 5.000 & $24,1 \mathrm{~ns}$ & $20,5 \mathrm{~ns}$ & $25,4 \mathrm{~ns}$ & $25,4 \quad b$ & 27,5 ns & $26,7 \mathrm{~ns}$ & $27,9 \mathrm{~ns}$ & $26,5 \mathrm{~ns}$ & $28,0 \mathrm{~ns}$ \\
\hline 7.500 & $31,0 \mathrm{~ns}$ & $25,6 \mathrm{~ns}$ & $36,6 \mathrm{~ns}$ & $27,6 \mathrm{ab}$ & $25,6 \mathrm{~ns}$ & $25,8 \mathrm{~ns}$ & $27,8 \mathrm{~ns}$ & $27,0 \mathrm{~ns}$ & $26,6 \mathrm{~ns}$ \\
\hline 10.000 & 30,2 ns & 22,9 ns & $31,9 \mathrm{~ns}$ & 33,8 a & 30,4 ns & $28,2 \mathrm{~ns}$ & $27,6 \mathrm{~ns}$ & $30,0 \mathrm{~ns}$ & $31,8 \mathrm{~ns}$ \\
\hline \multicolumn{10}{|c|}{ Profundidad 20-40 cm } \\
\hline A.melanoxylon & & $15,1 \mathrm{~ns}$ & 18,3 ns & $20,0 \mathrm{~ns}$ & 19,9 ns & $21,2 \mathrm{~ns}$ & $19,9 \mathrm{~ns}$ & 17,6 b & $18,7 \mathrm{~ns}$ \\
\hline E.globulus & & $18,2 \mathrm{~ns}$ & $26,8 \mathrm{~ns}$ & 24,2 ns & $24,1 \mathrm{~ns}$ & 24,5 ns & $21,8 \mathrm{~ns}$ & 23,6 a & $23,3 \mathrm{~ns}$ \\
\hline E.nitens & & $18,0 \mathrm{~ns}$ & $26,9 \mathrm{~ns}$ & 22,7 ns & $21,2 \mathrm{~ns}$ & $27,0 \mathrm{~ns}$ & $24,5 \mathrm{~ns}$ & 24,7 a & $23,2 \mathrm{~ns}$ \\
\hline
\end{tabular}

Revista Árvore, Viçosa-MG, v.37, n.6, p.1135-1144, 2013 

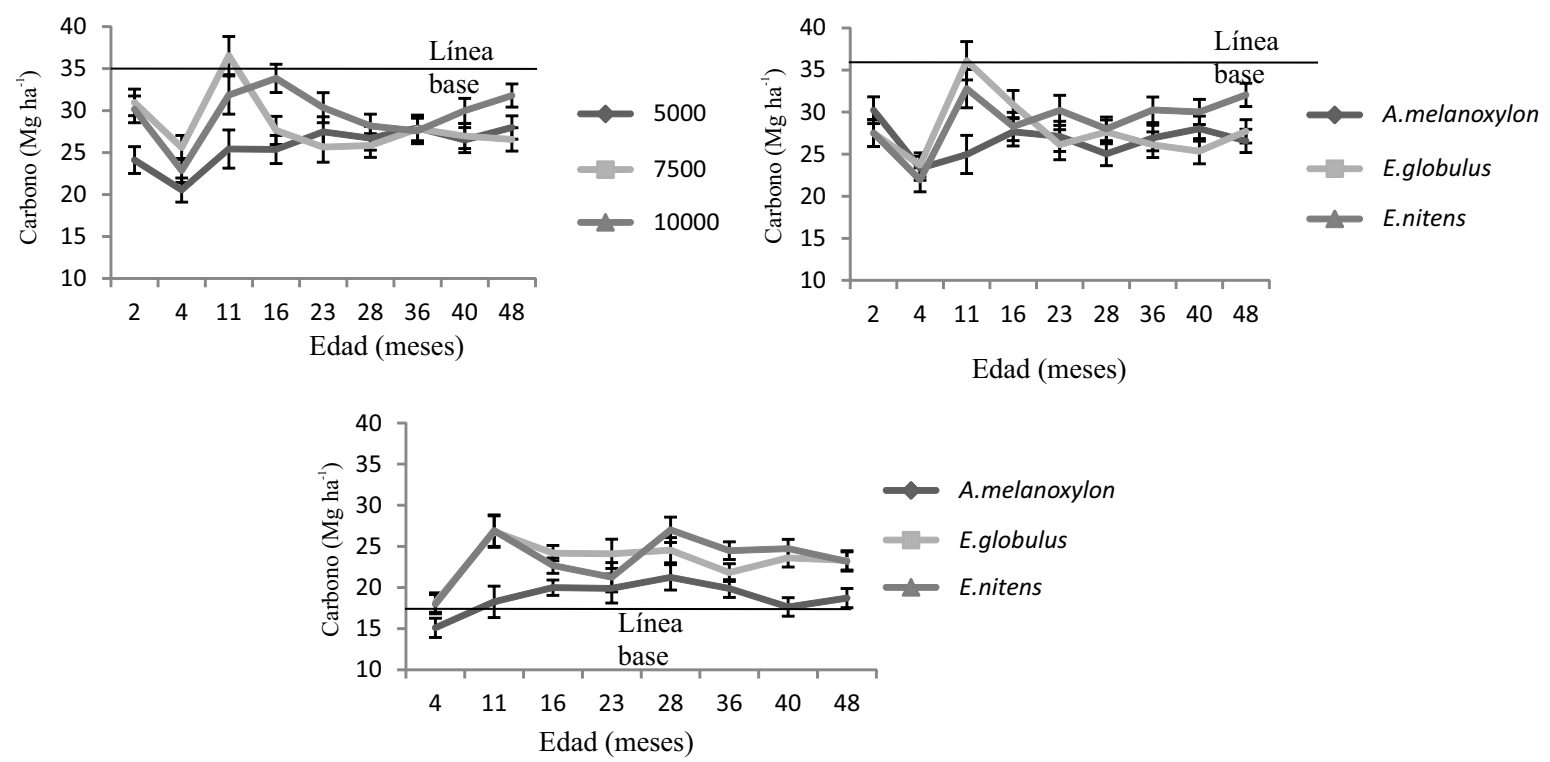

Figura 2 - Promedios para los efectos significativos de contenido de carbono ( $\left.\mathrm{Mg} \mathrm{ha}^{-1}\right)$ en las todas las densidades (5.000, 7.500 y 10.000 árboles ha-1 $^{-1}$ para todas la especies (Acacia melanoxylon, Eucalyptus globulus y E. nitens) a las profundidades 0-20 y 20-40 cm, en el sitio de suelos arenales. Barras de error corresponden al error estándar.

Figura 2 - Valores médios dos efeitos significativos do conteúdo de carbono ( $\mathrm{Mg} \mathrm{ha}^{-1}$ ) em todas as densidades (5.000, 7.500 e 10.000 árvores ha ${ }^{-1}$ ) em todas as espécies (Acacia melanoxylon, Eucalyptus globulus e E. nitens), às profundidades de 0-20 e 20-40 cm, no sítio de solos arenosos. As barras de erro correspondem ao erro-padrão.

rápidamente luego de la corta de los árboles. En este caso la caída de carbono solamente ocurrió hasta los 2 meses de edad de las plantaciones (7 meses postcosecha), lo que sugiere una capacidad de disminución de pérdidas de carbono por parte de estas plantaciones. Sin embargo, en la Figura 2 se puede apreciar un aumento de los contenidos de carbono en el suelo arenales en la profundidad 20 - $40 \mathrm{~cm}$ con respecto al a línea base. Esto puede deberse a la poca capacidad de este tipo de suelo en la retención de carbono, el cual puede lixiviarse a mayores profundidades rápidamente. Algunos autores han interpretado estos cambios debido a "el transporte del material masticado desde profundidades superiores por parte de la fauna del suelo, y la infusión de la materia orgánica disuelta de las profundidades superiores y, sobre todo, si la alteración de la descomposición acelerada de la labranza de la materia orgánica del suelo” (SANCHEZ et al., 2007), lo que podría verse reflejado en disminución de las profundidades superiores de suelo, como se observa en este caso en la Figura 3, donde existe una disminución con respecto a la línea base.

Las pérdidas en el carbono podrían atribuirse también a los cambios en el suelo realizados con la preparación de terreno. En las plantaciones forestales, parte del carbono captado quedan en el sitio formando parte del piso forestal, la no mecanización del suelo protege al mismo de la erosión y permite aumentar el monto de carbono secuestrado (CARROLL; SOMERVILLE, 2009). Mediante el manejo adecuado de la plantación, el carbono enterrado en el sistema radicular se mantendrá pudiendo ser este el mecanismo preferido para aumentar el carbono secuestrado a largo plazo (SANCHEZ et al. 2007). En este caso, posterior a la cosecha, se procedió a la eliminación de los tocones y a la preparación del terreno, lo que aumentaría las pérdidas de carbono, en el suelo; estas labores se realizaron con el fin de lograr un sitio lo más homogéneo posible en cuanto a la magnitud de carbono acumulado.

Los niveles de carbono total cambiaron en cada tratamiento en el tiempo, sin que esto represente una pérdida de carbono total a nivel de suelo de los 2 a los 48 meses después de establecida la plantación, lo que demuestra la capacidad que tiene estas plantaciones para mantener los niveles de carbono se suelo. Algunas investigaciones sugieren que esto podría deberse al rápido crecimiento radical y a la incorporación temprana de necromasa al piso forestal. Investigaciones previas han demostrado en plantaciones 
dendroenergéticas aumentos de carbono a nivel de suelo de hasta $1,6 \mathrm{Mg} \mathrm{C} \mathrm{ha-1}^{-1} \mathrm{año}^{-1}$, atribuyendo los aumentos en el carbono del suelo también al rápido crecimiento de los cultivos dendroenergéticos (SARTORI et al., 2007).

“La evaluación del carbono secuestrado en el suelo presenta dificultades generales debido a su natural variabilidad, lo que dificulta generalizar los beneficios de las prácticas agrícolas como la cero labranza que aumentan los niveles de carbono del suelo" (COLEMAN et al., 2004). En este caso todos los muestreos fueron de muestras compuestas para minimizar los efectos de esta variación, por lo que los datos presentados muestran que los sitios presentan dinámicas diferentes. Esto concuerda con lo mencionado por BARAL: GUHA (2004), que indican que el monto captado de carbono por el suelo es altamente variable y específico de cada sitio. Esa especificidad mencionada puede comprobarse al observar en las Tablas 2 y 3, pues los montos de carbono variaron entre los sitios efecto de la diferencia en el tipo de suelo entre los sitios.

En plantaciones dendroenergéticas, Sanchez et al. (2007) evidenciaron que los niveles de carbono precosecha se mantuvieron durante el primer año, atribuido a la rápida incorporación de la necromasa; no fue sino a los 3 años que logran evidenciar una disminución. Por otra parte, Coleman et al. (2004) no encontraron diferencias en los años iniciales en los niveles de carbono de suelo, encontrando pequeños incrementos a los 40 meses de la plantación.

Lo anterior está relacionado con el mantenimiento de los niveles de carbono desde los dos meses hasta los 48 meses de edad mostrado en este ensayo, solamente en el suelo granítico, las especies E. camaldulensis y E. nitens ambas en la densidad de 7.500 árboles ha ${ }^{-1}$ disminuyeron el contenido de carbono del suelo, sin embargo, esta disminución se evidenció en los últimos 8 meses en ambos casos (ver Tabla 2). Mann y Tolbert (2000) evidenciaron la capacidad que estas plantaciones tiene en aumentar la cantidad de carbono del suelo. Esto podría explicar la capacidad que mostraron los tratamientos aplicados en la conservación del carbono desde los 2 meses hasta los 48 meses.

Por otra parte, existe una relación estrecha entre los niveles de materia orgánica y los niveles nutricionales, así como beneficios en las propiedades físicas como densidad del suelo y resistencia (SANCHEZ et al., 2003).
En este caso, los tratamientos presentaron diferencias en los contenidos de biomasa aérea (SANDOVAL, 2012), lo que podría indicar diferencias en los contenidos nutricionales, sin embargo, no se ha logrado diferenciar efectos en el carbono del suelo mineral entre los tratamientos. Lo anterior podría deberse a que las diferentes especies y densidades ensayadas no lograron una cobertura del suelo a la misma edad; además de ello, no se logró relacionar los niveles de carbono del suelo con los posibles efectos de fijación de nitrógeno de la especie leguminosa (A. melanoxylon) y la no fijación de los eucaliptos en ninguno de los sitios.

\section{CONCLUSIÓN}

Se evidenciaron cambios a lo largo del tiempo, lo que demuestra que a pesar de la variabilidad que presenta el carbono, la incorporación del mismo al suelo en el periodo evaluado puede compensar las pérdidas que ocurren posterior a la una cosecha forestal a partir de los 2 meses de establecidos los árboles.

Sin embargo, no es claro aun cuál es la especie o densidad que puede maximizar esta vía de secuestro de carbono. Esto motiva a continuar con el estudio de estas plantaciones, con el fin de comprender los mecanismos que tienen para la reducción del carbono atmosférico mediante la captura y almacenamiento de carbono a nivel de suelo y como este puede verse influenciado.

\section{RECONOCIMIENTO}

Al programa de doctorado de la Facultad de Ciencias Forestales de la Universidad de Concepción, al Proyecto INNOVA Bíobío N 06-PC S1-33 por el financiamiento; al Programa de Becas para Estudios de Doctorado para Estudiantes Extranjeros en Chile de CONICYT, Chile; a la beca para estudios de postgrado de la OEA y a la beca del Programa de Formación de Recurso Humano en Ciencia y Tecnología del MICIT, Costa Rica. Al Laboratorio de Suelos Nutrición y Productividad Forestal, y a la Cooperativa de Producción Forestal y a los compañeros por su colaboración.

\section{REFERENCIAS}

ADLER, P. R.; GROSSO, S. J. D.; PARTON, W. J. Life cycle assessment of net greenhouse-gas flux for bioenergy cropping systems. Ecological Applications, v.17, n.3, p.675-691, 2007. 
ANDERSEN, R. S.; TOWERS, W.; SMITH, P. Assessing the potential for biomass energy to contribute to Scotland's renewable energy needs. Biomass \& Bioenergy, v.29, n.2, p.73-82, 2005.

BARAL, A.; GUHA, G. S. Trees for carbon sequestration or fossil fuel substitution: the issue of cost vs. carbon benefit. Biomass \& Bioenergy, v.27, n.1, p.41-55, 2004.

BENITES, V. D. M. et al. Análise discriminante de solos sob diferentes usos em área de Mata Atlântica a partir de atributos da matéria orgânica. Revista Árbore, v.34, n.4, p.685-690, 2010.

BERNDES, G.; HOOGWIJK, M.; van den BROEK, R. The contribution of biomass in the future global energy supply: a review of 17 studies. Biomass \& Bioenergy, v.25, n.1, p.1-28, 2003.

CARROLL, A.; SOMERVILLE, C. Cellulosic Biofuels. Annual Review of Plant Biology, v.60, p.165-182, 2009.

COLEMAN, M. D. et al. Comparing soil carbon of short rotation poplar plantations with agricultural crops and woodlots in North Central United States. Environmental Management, v.33, p.S299-S308, 2004.

ERICSSON, E. Carbon accumulation and fossil fuel substitution during different rotation scenarios. Scandinavian Journal of Forest Research, v.18, n.3, p.269, 2003.

FUNG, P. Y. H. et al. The potential for bioenergy production from Australian forests, its contribution to national greenhouse targets and recent developments in conversion processes. Biomass \& Bioenergy, v.22, n.4, p.223-236, 2002.

KARP, A.; SHIELD, I. Bioenergy from plants and the sustainable yield challenge. New

Phytologist, v.179, n.1, p.15-32, 2008.

MAFRA, Á. L. et al. Carbono orgânico e atributos químicos do solo em áreas florestais. Revista Árbore, v.32, n.2, p.217-224, 2008.
MAGDOFF, F. Soil organic matter fractions and implications for interpreting organic matter test. In: MAGDOFF F. M. T.; HANLON, E. A. (Ed.). Soil organic matter: analysis and interpretation. Wisconsin: SSSA Special Publication, 1996. v.46. p.11-20.

MANN, L.; TOLBERT, V. Soil Sustainability in renewable biomass plantings. Ambio, v.29, n.8, p.492-498, 2000.

MERINO, A. et al. Soil properties in a hilly area following different harvesting management practices. Forest Ecology and Management, v.103, n.2-3, p.235-246, 1998.

POWERS, R. F. et al. The North American longterm soil productivity experiment: Findings from the first decade of research. Forest Ecology and Management, v.220, n.1-3, p.31-50, 2005.

SANCHEZ, F. G.; CARTER, E. A.; KLEPAC, J. F. Enhancing the soil organic matter pool through biomass incorporation. Biomass \& Bioenergy, v.24, n.4-5, p.337-349, 2003.

SANCHEZ, F. G. et al. Soil carbon, after 3 years, under short-rotation woody crops grown under varying nutrient and water availability. Biomass \& Bioenergy, v.31, n.11-12, p.793-801, 2007.

SANDOVAL, S. P. Modelación del crecimiento y rendimiento en biomasa aérea de ensayos dendroenergéticos. 2012. p.185. Tese (Doctor) - Facultad de Ciencias Forestales, Universidad de Concepción, Concepción Chile, 2012.

SARTORI, F. et al. Changes in soil carbon and nutrient pools along a chronosequence of poplar plantations in the Columbia Plateau, Oregon, USA. Agriculture Ecosystems \& Environment, v.122, n.3, p.325-339, 2007.

SARTORI, F. et al. Potential soil carbon sequestration and $\mathrm{CO} 2$ offset by dedicated energy crops in the USA. Critical Reviews in Plant Sciences, v.25, n.5, p.441-472, 2006.

SAS-INSTITUTE-INC. SAS/STAT User's guide: Version 8. Cary: 2000.

Revista Árvore, Viçosa-MG, v.37, n.6, p.1135-1144, 2013 
SLESAK, R. A.; SCHOENHOLTZ, S. H.;

HARRINGTON, T. B. Soil carbon and nutrient pools in Douglas-fir plantations 5 years after manipulating biomass and competing vegetation in the Pacific Northwest. Forest Ecology and Management, v.262, n.9, p.1722-1728, 2011.

STUPAK, I. et al. Sustainable utilisation of forest biomass for energy-possibilities and problems: Policy, legislation, certification, and recommendations and guidelines in the Nordic, Baltic, and other European countries. Biomass \& Bioenergy, v.31, n.10, p.666-684, 2007.
TABATABAI, M. Soil organic matter testing: an overview. In: MAGDOFF F. M. T.; HANLON, E. A. (Ed.).Soil organic matter: analysis and interpretation. Wisconsin: SSSA Special Publication, 1996. v.46. p.1-9.

USSIRI, D. A. N.; JOHNSON, C. E. Organic matter composition and dynamics in a northern hardwood forest ecosystem 15 years after clear-cutting. Forest Ecology and Management, v.240, n.1-3, p.131-142, 2007. 